\title{
Congruences for partition functions related to mock theta functions
}

\author{
Shane Chern and Li-Jun Hao
}

\begin{abstract}
Partitions associated with mock theta functions have received a great deal of attention in the literature. Recently, Choi and Kim derived several partition identities from the third and sixth order mock theta functions. In addition, three Ramanujan-type congruences were established by them. In this paper, we present some new congruences for these partition functions.
\end{abstract}

Keywords. Partition, $t$-core partition, cubic partition, mock theta function, Ramanujantype congruence.

2010MSC. 11P83, 05A17.

\section{Introduction}

A partition of a positive integer $n$ is a finite nonincreasing sequence of positive integers whose sum equals $n$. Furthermore, a partition is called a $t$-core partition if there are no hook numbers being multiples of $t$. Let $a_{t}(n)$ be the number of $t$-core partitions of $n$. It is known [17] that

$$
\sum_{n=0}^{\infty} a_{t}(n) q^{n}=\frac{\left(q^{t} ; q^{t}\right)_{\infty}^{t}}{(q ; q)_{\infty}}
$$

Here and in what follows, we make use of the standard $q$-series notation (cf. [18]).

$$
\begin{aligned}
(a)_{n}=(a ; q)_{n} & :=\prod_{k=0}^{n-1}\left(1-a q^{k}\right), \\
(a)_{\infty}=(a ; q)_{\infty} & :=\prod_{k=0}^{\infty}\left(1-a q^{k}\right), \\
\left(a_{1}, a_{2}, \cdots, a_{m} ; q\right)_{\infty} & :=\left(a_{1} ; q\right)_{\infty}\left(a_{2} ; q\right)_{\infty} \cdots\left(a_{m} ; q\right)_{\infty} .
\end{aligned}
$$

In addition, the cubic partition, which was introduced by Chan [11, 12] and named by Kim [20] in connection with Ramanujan's cubic continued fractions, is a 2-color partition where the second color appears only in multiples of 2. Let $a(n)$ denote the number of cubic partitions of $n$, then its generating function is

$$
\sum_{n=0}^{\infty} a(n) q^{n}=\frac{1}{(q ; q)_{\infty}\left(q^{2} ; q^{2}\right)_{\infty}} .
$$

In his last letter to Hardy [9, pp. 220-223], Ramanujan defined 17 functions, which he called mock theta functions. Since then, there has been an intense study of partition interpretations for mock theta functions; see $[2,3,4,5,6]$. 
Recently, Choi and Kim [15] obtained the following identity related to the third order mock theta function,

$$
v(q)+v_{3}(q, q ; q)=2 \frac{\left(q^{4} ; q^{4}\right)_{\infty}^{3}}{\left(q^{2} ; q^{2}\right)_{\infty}^{2}}
$$

where $v(q)$ is the third mock theta function and $v_{3}(q, q ; q)$ is defined by Choi [14],

$$
v(q)=\sum_{n=0}^{\infty} \frac{q^{n(n+1)}}{\left(-q ; q^{2}\right)_{n+1}}, \quad v_{3}(q, q ; q)=\sum_{n=0}^{\infty} q^{n}\left(-q ; q^{2}\right)_{n}
$$

They also gave the following identities related to the sixth order mock theta functions,

$$
\begin{aligned}
\Psi(q)+2 \Psi_{-}(q) & =3 \frac{q\left(q^{6} ; q^{6}\right)_{\infty}^{3}}{(q ; q)_{\infty}\left(q^{2} ; q^{2}\right)_{\infty}} \\
2 \rho(q)+\lambda(q) & =3 \frac{\left(q^{3} ; q^{3}\right)_{\infty}^{3}}{(q ; q)_{\infty}\left(q^{2} ; q^{2}\right)_{\infty}}
\end{aligned}
$$

where $\Psi(q), \Psi_{-}(q), \rho(q)$ and $\lambda(q)$ are the sixth order mock theta functions,

$$
\begin{array}{ll}
\Psi(q)=\sum_{n=0}^{\infty} \frac{(-1)^{n} q^{(n+1)^{2}}\left(q ; q^{2}\right)_{n}}{(-q ; q)_{2 n+1}}, & \Psi_{-}(q)=\sum_{n=1}^{\infty} \frac{q^{n}(-q ; q)_{2 n-2}}{\left(q ; q^{2}\right)_{n}} \\
\rho(q)=\sum_{n=0}^{\infty} \frac{q^{\left(\begin{array}{c}
n+1 \\
2
\end{array}\right)(-q ; q)_{n}}}{\left(q ; q^{2}\right)_{n+1}}, & \lambda(q)=\sum_{n=0}^{\infty} \frac{(-1)^{n} q^{n}\left(q ; q^{2}\right)_{n}}{(-q ; q)_{n}}
\end{array}
$$

Meanwhile, they studied three analogous partition functions defined by

$$
\begin{aligned}
\sum_{n=0}^{\infty} b(n) q^{n} & =\frac{\left(q^{4} ; q^{4}\right)_{\infty}^{3}}{\left(q^{2} ; q^{2}\right)_{\infty}^{2}} \\
\sum_{n=0}^{\infty} c(n) q^{n} & =\frac{q\left(q^{6} ; q^{6}\right)_{\infty}^{3}}{(q ; q)_{\infty}\left(q^{2} ; q^{2}\right)_{\infty}} \\
\sum_{n=0}^{\infty} d(n) q^{n} & =\frac{\left(q^{3} ; q^{3}\right)_{\infty}^{3}}{(q ; q)_{\infty}\left(q^{2} ; q^{2}\right)_{\infty}}
\end{aligned}
$$

where $b(n)$ denotes the number of partition pairs $(\lambda, \sigma)$ where $\sigma$ is a partition into distinct even parts and $\lambda$ is a partition into even parts of which 2-modular diagram is 2-core, and both $c(n)$ and $d(n)$ can be regarded as 3-core cubic partitions.

In this paper, we mainly study Ramanujan-type congruences for these partition functions. This paper is organized as follows. In Sect. 2, we introduce some preliminary results. In the next two sections, we will prove some Ramanujan-type congruences for $b(n)$ and $c(n)$, respectively. In Sect. 5, by employing $p$-dissection formulas of Ramanujan's theta functions $\psi(q)$ and $f(-q)$ established by Cui and $\mathrm{Gu}[16]$ as well as $(p, k)$-parameter representations due to Alaca and Williams [1], we show some congruences for $d(n)$. Finally, we end this paper with several open problems. 


\section{Preliminaries}

Let $f(a, b)$ be Ramanujan's general theta function given by

$$
f(a, b)=\sum_{n=-\infty}^{\infty} a^{\frac{n(n+1)}{2}} b^{\frac{n(n-1)}{2}}, \quad|a b|<1 .
$$

We now introduce the following Ramanujan's classical theta functions,

$$
\begin{aligned}
\varphi(q) & :=f(q, q)=\sum_{n=-\infty}^{\infty} q^{n^{2}}=\frac{f_{2}^{5}}{f_{1}^{2} f_{4}^{2}}, \\
\psi(q) & :=f\left(q, q^{3}\right)=\sum_{n=0}^{\infty} q^{\frac{n(n+1)}{2}}=\frac{f_{2}^{2}}{f_{1}}, \\
f(-q) & :=f\left(-q,-q^{2}\right)=\sum_{n=-\infty}^{\infty}(-1)^{n} q^{\frac{n(3 n+1)}{2}}=f_{1} .
\end{aligned}
$$

One readily verifies

$$
\varphi(-q)=\frac{f_{1}^{2}}{f_{2}}
$$

Here and in the sequel, we write $f_{k}:=\left(q^{k} ; q^{k}\right)_{\infty}$ for positive integers $k$ for convenience.

We first require the following 2-dissections.

Lemma 2.1. It holds that

$$
\begin{aligned}
& \frac{1}{f_{1}^{2}}=\frac{f_{8}^{5}}{f_{2}^{5} f_{16}^{2}}+2 q \frac{f_{4}^{2} f_{16}^{2}}{f_{2}^{5} f_{8}}, \\
& \frac{f_{3}}{f_{1}^{3}}=\frac{f_{4}^{6} f_{6}^{3}}{f_{2}^{9} f_{12}^{2}}+3 q \frac{f_{4}^{2} f_{6} f_{12}^{2}}{f_{2}^{7}}, \\
& \frac{f_{3}^{3}}{f_{1}}=\frac{f_{4}^{3} f_{6}^{2}}{f_{2}^{2} f_{12}}+q \frac{f_{12}^{3}}{f_{4}} .
\end{aligned}
$$

Proof. Here (2.5) comes from the 2-dissection of $\varphi(q)$ (cf. [8, p. 40, Entry 25]). For (2.6) and (2.7), see [25].

The following 3-dissections are also necessary.

Lemma 2.2. It holds that

$$
\begin{aligned}
\frac{1}{\varphi(-q)} & =\frac{\varphi^{3}\left(-q^{9}\right)}{\varphi^{4}\left(-q^{3}\right)}\left(1+2 q w\left(q^{3}\right)+4 q^{2} w^{2}\left(q^{3}\right)\right), \\
\frac{1}{\psi(q)} & =\frac{\psi^{3}\left(q^{9}\right)}{\psi^{4}\left(q^{3}\right)}\left(\frac{1}{w^{2}\left(q^{3}\right)}-\frac{q}{w\left(q^{3}\right)}+q^{2}\right),
\end{aligned}
$$

where

Furthermore,

$$
w(q)=\frac{f_{1} f_{6}^{3}}{f_{2} f_{3}^{3}} .
$$

where

$$
\frac{1}{f_{1}^{3}}=\frac{f_{9}^{3}}{f_{3}^{12}}\left(P^{2}\left(q^{3}\right)+3 q P\left(q^{3}\right) f_{9}^{3}+9 q^{2} f_{9}^{6}\right)
$$

$$
P(q)=f_{1}\left(\frac{\varphi^{3}\left(-q^{3}\right)}{\varphi(-q)}+4 q \frac{\psi^{3}\left(q^{3}\right)}{\psi(q)}\right)
$$


Proof. For (2.8) and (2.9), see Baruah and Ojah [7]. For (2.11), see Wang [23]. Note that Wang [23] showed

$$
P(q)=f_{1}\left(1+6 \sum_{n \geq 0}\left(\frac{q^{3 n+1}}{1-q^{3 n+1}}-\frac{q^{3 n+2}}{1-q^{3 n+2}}\right)\right) .
$$

We know from [22, Eqs. (3.2) and (3.5)] that

$$
\begin{gathered}
4 q \frac{\psi^{3}\left(q^{3}\right)}{\psi(q)}=4 \sum_{n \geq 0}\left(\frac{q^{3 n+1}}{1-q^{6 n+2}}-\frac{q^{3 n+2}}{1-q^{6 n+4}}\right), \\
\frac{\varphi^{3}\left(-q^{3}\right)}{\varphi(-q)}=1+2 \sum_{n \geq 0}\left(\frac{q^{6 n+1}}{1-q^{6 n+1}}+\frac{q^{6 n+2}}{1-q^{6 n+2}}-\frac{q^{6 n+4}}{1-q^{6 n+4}}-\frac{q^{6 n+5}}{1-q^{6 n+5}}\right) .
\end{gathered}
$$

Hence (2.12) follows immediately by the following trivial identity

$$
\frac{x}{1-x^{2}}=\frac{x}{1-x}-\frac{x^{2}}{1-x^{2}} .
$$

Furthermore, we need

Lemma 2.3 ([16, Theorem 2.1]). For any odd prime $p$,

$$
\psi(q)=q^{\frac{p^{2}-1}{8}} \psi\left(q^{p^{2}}\right)+\sum_{k=0}^{\frac{p-3}{2}} q^{\frac{k^{2}+k}{2}} f\left(q^{\frac{p^{2}+(2 k+1) p}{2}}, q^{\frac{p^{2}-(2 k+1) p}{2}}\right) .
$$

Furthermore, we claim that for $0 \leq k \leq(p-3) / 2$,

$$
\frac{k^{2}+k}{2} \not \equiv \frac{p^{2}-1}{8} \quad(\bmod p) \text {. }
$$

Lemma 2.4 ([16, Theorem 2.2]). For any prime $p \geq 5$,

$$
\begin{aligned}
f(-q)=(-1)^{\frac{ \pm p-1}{6}} q^{\frac{p^{2}-1}{24}} f\left(-q^{p^{2}}\right) & \\
& +\sum_{\substack{k=-\frac{p-1}{2} \\
k \neq \frac{ \pm p-1}{6}}}^{\frac{p-1}{2}}(-1)^{k} q^{\frac{3 k^{2}+k}{2}} f\left(-q^{\frac{3 p^{2}+(6 k+1) p}{2}},-q^{\frac{3 p^{2}-(6 k+1) p}{2}}\right) .
\end{aligned}
$$

Furthermore, we claim that for $-(p-1) / 2 \leq k \leq(p-1) / 2$ and $k \neq( \pm p-1) / 6$,

$$
\frac{3 k^{2}+k}{2} \not \equiv \frac{p^{2}-1}{24} \quad(\bmod p) .
$$

Here for any prime $p \geq 5$,

$$
\frac{ \pm p-1}{6}:=\left\{\begin{aligned}
\frac{p-1}{6}, & p \equiv 1 \quad(\bmod 6) \\
\frac{-p-1}{6}, & p \equiv-1 \quad(\bmod 6) .
\end{aligned}\right.
$$

At last, we require the following relations due to Alaca and Williams [1]. 
Lemma 2.5. Let

$$
p=p(q):=\frac{\varphi^{2}(q)-\varphi^{2}\left(q^{3}\right)}{2 \varphi^{2}\left(q^{3}\right)}
$$

and

$$
k=k(q):=\frac{\varphi^{3}\left(q^{3}\right)}{\varphi(q)} .
$$

Then

$$
\begin{aligned}
f_{1} & =2^{-\frac{1}{6}} q^{-\frac{1}{24}} p^{\frac{1}{24}}(1-p)^{\frac{1}{2}}(1+p)^{\frac{1}{6}}(1+2 p)^{\frac{1}{8}}(2+p)^{\frac{1}{8}} k^{\frac{1}{2}}, \\
f_{2} & =2^{-\frac{1}{3}} q^{-\frac{1}{12}} p^{\frac{1}{12}}(1-p)^{\frac{1}{4}}(1+p)^{\frac{1}{12}}(1+2 p)^{\frac{1}{4}}(2+p)^{\frac{1}{4}} k^{\frac{1}{2}}, \\
f_{3} & =2^{-\frac{1}{6}} q^{-\frac{1}{8}} p^{\frac{1}{8}}(1-p)^{\frac{1}{6}}(1+p)^{\frac{1}{2}}(1+2 p)^{\frac{1}{24}}(2+p)^{\frac{1}{24}} k^{\frac{1}{2}}, \\
f_{4} & =2^{-\frac{2}{3}} q^{-\frac{1}{6}} p^{\frac{1}{6}}(1-p)^{\frac{1}{8}}(1+p)^{\frac{1}{24}}(1+2 p)^{\frac{1}{8}}(2+p)^{\frac{1}{2}} k^{\frac{1}{2}}, \\
f_{6} & =2^{-\frac{1}{3}} q^{-\frac{1}{4}} p^{\frac{1}{4}}(1-p)^{\frac{1}{12}}(1+p)^{\frac{1}{4}}(1+2 p)^{\frac{1}{12}}(2+p)^{\frac{1}{12}} k^{\frac{1}{2}}, \\
f_{12} & =2^{-\frac{2}{3}} q^{-\frac{1}{2}} p^{\frac{1}{2}}(1-p)^{\frac{1}{24}}(1+p)^{\frac{1}{8}}(1+2 p)^{\frac{1}{24}}(2+p)^{\frac{1}{6}} k^{\frac{1}{2}} .
\end{aligned}
$$

\section{Congruences for $b(n)$}

Theorem 3.1. For $n \geq 0, \alpha \geq 1$, and prime $p \geq 5$, we have

$$
b\left(p^{2 \alpha} n+\frac{(3 j+p) p^{2 \alpha-1}-1}{3}\right) \equiv 0 \quad(\bmod 2),
$$

where $j=1,2, \cdots, p-1$.

Proof. In light of (1.1), we derive that

$$
\sum_{n=0}^{\infty} b(n) q^{n}=\frac{f_{4}^{3}}{f_{2}^{2}} \equiv f_{8} \quad(\bmod 2) .
$$

Applying Lemma 2.4, we deduce that, for any prime $p \geq 5$,

$$
\sum_{n=0}^{\infty} b\left(p n+\frac{p^{2}-1}{3}\right) q^{n} \equiv(-1)^{\frac{ \pm p-1}{6}} f\left(-q^{8 p}\right) \quad(\bmod 2),
$$

and

Moreover,

$$
\sum_{n=0}^{\infty} b\left(p^{2} n+\frac{p^{2}-1}{3}\right) q^{n} \equiv(-1)^{\frac{ \pm p-1}{6}} f\left(-q^{8}\right) \quad(\bmod 2) .
$$

$$
\sum_{n=0}^{\infty} b\left(p^{3} n+\frac{p^{4}-1}{3}\right) q^{n} \equiv f\left(-q^{8 p}\right) \quad(\bmod 2) .
$$

Hence, by induction on $\alpha$, we derive that, for $\alpha \geq 1$,

$$
\sum_{n=0}^{\infty} b\left(p^{2 \alpha-1} n+\frac{p^{2 \alpha}-1}{3}\right) q^{n} \equiv(-1)^{\alpha\left(\frac{ \pm p-1}{6}\right)} f\left(-q^{8 p}\right) \quad(\bmod 2) .
$$

This immediately leads to

$$
b\left(p^{2 \alpha-1}(p n+j)+\frac{p^{2 \alpha}-1}{3}\right) \equiv 0 \quad(\bmod 2),
$$

for $j=1,2, \cdots, p-1$. We complete the proof. 
Remark 3.1. When studying 1-shell totally symmetric plane partition function $f(n)$ (which is different to Ramanujan's theta function $f(-q)$ given in Sect. 2) introduced by Blecher [10], Hirschhorn and Sellers [19] proved that, for $n \geq 1$,

$$
f(3 n-2)=h(n),
$$

with

$$
\sum_{n=0}^{\infty} h(2 n+1) q^{n}=\frac{f_{2}^{3}}{f_{1}^{2}}
$$

A couple of congruences modulo powers of 2 and 5 for $h(n)$ have been obtained subsequently; see $[13,24,26]$. We see from (1.1) that

$$
b(2 n)=h(2 n+1) .
$$

One therefore may obtain some congruences for $b(n)$ as well. For example,

$$
b(8 n+6) \equiv 0 \quad(\bmod 4) .
$$

\section{Congruences for $c(n)$}

Theorem 4.1. For $n \geq 0$, we have

$$
c(27 n+24) \equiv 0 \quad(\bmod 9) .
$$

Proof. We see from (1.2) and Lemma 2.2 that

$$
\begin{aligned}
\sum_{n=0}^{\infty} c(n) q^{n}= & \frac{q f_{6}^{3}}{\varphi(-q) \psi(q)} \\
= & q f_{6}^{3} \frac{\varphi^{3}\left(-q^{9}\right) \psi^{3}\left(q^{9}\right)}{\varphi^{4}\left(-q^{3}\right) \psi^{4}\left(q^{3}\right)}\left(1+2 q w\left(q^{3}\right)+4 q^{2} w^{2}\left(q^{3}\right)\right) \\
& \times\left(\frac{1}{w^{2}\left(q^{3}\right)}-\frac{q}{w\left(q^{3}\right)}+q^{2}\right) .
\end{aligned}
$$

Employing Lemma 2.2, we deduce that

$$
\begin{aligned}
\sum_{n=0}^{\infty} c(3 n) q^{n}= & \frac{3 q \varphi^{3}\left(-q^{3}\right) \psi^{3}\left(q^{3}\right)}{f_{1}^{3} \varphi(-q) \psi(q)} \\
= & \frac{3 q \varphi^{3}\left(-q^{9}\right) \psi^{3}\left(q^{9}\right) f_{9}^{3}}{\varphi\left(-q^{3}\right) \psi\left(q^{3}\right) f_{3}^{12}}\left(P^{2}\left(q^{3}\right)+3 q P\left(q^{3}\right) f_{9}^{3}+9 q^{2} f_{9}^{6}\right) \\
& \quad \times\left(1+2 q w\left(q^{3}\right)+4 q^{2} w^{2}\left(q^{3}\right)\right)\left(\frac{1}{w^{2}\left(q^{3}\right)}-\frac{q}{w\left(q^{3}\right)}+q^{2}\right)
\end{aligned}
$$

Extracting terms involving $q^{3 n+2}$ and replacing $q^{3}$ by $q$ in (4.2), it follows that

$$
\sum_{n=0}^{\infty} c(9 n+6) q^{n}=12 \frac{f_{2}^{2} f_{3}^{21}}{f_{1}^{16} f_{6}^{6}}+135 q \frac{f_{3}^{12} f_{6}^{3}}{f_{1}^{13} f_{2}}+72 q^{2} \frac{f_{3}^{3} f_{6}^{12}}{f_{1}^{10} f_{2}^{4}}+192 q^{3} \frac{f_{6}^{21}}{f_{1}^{7} f_{2}^{7} f_{3}^{6}} .
$$

Hence,

$$
\begin{aligned}
\sum_{n=0}^{\infty} c(9 n+6) q^{n} & \equiv 3 \frac{f_{2}^{2} f_{3}^{21}}{f_{1}^{16} f_{6}^{6}}+3 q^{3} \frac{f_{6}^{21}}{f_{1}^{7} f_{2}^{7} f_{3}^{6}} \\
& \equiv 3 \frac{f_{2}^{2}}{f_{1}}\left(\frac{f_{3}^{16}}{f_{6}^{6}}+q^{3} \frac{f_{6}^{18}}{f_{3}^{8}}\right) \quad(\bmod 9)
\end{aligned}
$$


Noting that $f_{2}^{2} / f_{1}$ contains no terms of the form $q^{3 n+2}$, we have

$$
\sum_{n=0}^{\infty} c(27 n+24) q^{n} \equiv 0 \quad(\bmod 9)
$$

It therefore ends the proof.

Theorem 4.2. For $n \geq 0$, we have

$$
c(45 n+t) \equiv 0 \quad(\bmod 5),
$$

where $t=9$ and 18 .

Proof. Referring to (4.2), we have

$$
\sum_{n=0}^{\infty} c(9 n) q^{n}=45 q \frac{f_{2} f_{3}^{18}}{f_{1}^{15} f_{6}^{3}}+90 q^{2} \frac{f_{3}^{9} f_{6}^{6}}{f_{1}^{12} f_{2}^{2}}+288 q^{3} \frac{f_{6}^{15}}{f_{1}^{9} f_{2}^{5}} .
$$

Hence,

$$
\sum_{n=0}^{\infty} c(9 n) q^{n} \equiv 3 q^{3} f_{1} \frac{f_{30}^{3}}{f_{5}^{2} f_{10}} \quad(\bmod 5) .
$$

Since $f_{1}$ contains no terms of the form $q^{5 n+3}$ and $q^{5 n+4}$, we have

$$
c(9(5 n+1))=c(45 n+9) \equiv 0 \quad(\bmod 5),
$$

and

$$
c(9(5 n+2))=c(45 n+18) \equiv 0 \quad(\bmod 5) .
$$

This yields that (4.3).

Corollary 4.3. For $n \geq 0$, we have

$$
c(45 n+t) \equiv 0 \quad(\bmod 15)
$$

where $t=9$ and 18 .

Proof. We know from [15, Theorem 4.2] that

$$
c(3 n) \equiv 0 \quad(\bmod 3) .
$$

In fact, it is a direct consequence of (4.2). Hence, Corollary 4.3 follows by Theorem 4.2 .

\section{Congruences for $d(n)$}

Theorem 5.1. For $n \geq 0, \alpha \geq 1$, and prime $p \geq 3$,

$$
d\left(2 p^{2 \alpha}+\frac{(8 j+p) p^{2 \alpha-1}-1}{4}\right) \equiv 0 \quad(\bmod 2),
$$

where $j=1,2, \cdots, p-1$.

Proof. From (1.3), one can see

$$
\sum_{n=0}^{\infty} d(n) q^{n}=\frac{f_{3}^{3}}{f_{1} f_{2}} \equiv f_{6} \frac{f_{3}}{f_{1}^{3}} \quad(\bmod 2) .
$$

With the help of (2.6), we have

$$
\sum_{n=0}^{\infty} d(n) q^{n} \equiv \frac{f_{4}^{6} f_{6}^{4}}{f_{2}^{9} f_{12}^{2}}+3 q \frac{f_{4}^{2} f_{6}^{2} f_{12}^{2}}{f_{2}^{7}} \quad(\bmod 2) .
$$


Hence,

$$
\sum_{n=0}^{\infty} d(2 n) q^{n} \equiv \frac{f_{2}^{6} f_{3}^{4}}{f_{1}^{9} f_{6}^{2}} \equiv \psi(q) \quad(\bmod 2) .
$$

Invoking Lemma 2.3, for any odd prime $p$, we derive that

$$
\sum_{n=0}^{\infty} d\left(2\left(p n+\frac{p^{2}-1}{8}\right)\right) q^{n} \equiv \psi\left(q^{p}\right) \quad(\bmod 2),
$$

and

$$
\sum_{n=0}^{\infty} d\left(2\left(p^{2} n+\frac{p^{2}-1}{8}\right)\right) q^{n} \equiv \psi(q) \quad(\bmod 2) .
$$

Furthermore,

$$
\sum_{n=0}^{\infty} d\left(2 p^{3} n+\frac{p^{4}-1}{4}\right) q^{n} \equiv \psi\left(q^{p}\right) \quad(\bmod 2) .
$$

It therefore follows by induction on $\alpha$ that for $\alpha \geq 1$,

$$
\sum_{n=0}^{\infty} d\left(2 p^{2 \alpha-1} n+\frac{p^{2 \alpha}-1}{4}\right) q^{n} \equiv \psi\left(q^{p}\right) \quad(\bmod 2) .
$$

Thus, for $j=1,2, \cdots, p-1$,

$$
d\left(2 p^{2 \alpha-1}(p n+j)+\frac{p^{2 \alpha}-1}{4}\right) \equiv 0 \quad(\bmod 2),
$$

which is the desired result.

Theorem 5.2. For $n \geq 0, \alpha \geq 1$, and prime $p \geq 5$, we have

$$
d\left(6 p^{2 \alpha} n+\frac{(24 j+p) p^{2 \alpha-1}-1}{4}\right) \equiv 0 \quad(\bmod 3),
$$

where $j=1,2, \cdots, p-1$.

Proof. It follows by (2.8) and (2.9) that

$$
\begin{aligned}
\sum_{n=0}^{\infty} d(n)= & \frac{f_{3}^{3}}{\varphi(-q) \psi(q)} \\
= & f_{3}^{3} \frac{\varphi^{3}\left(-q^{9}\right) \psi^{3}\left(q^{9}\right)}{\varphi^{4}\left(-q^{3}\right) \psi^{4}\left(q^{3}\right)}\left(1+2 q w\left(q^{3}\right)+4 q^{2} w^{2}\left(q^{3}\right)\right) \\
& \times\left(\frac{1}{w^{2}\left(q^{3}\right)}-\frac{q}{w\left(q^{3}\right)}+q^{2}\right) .
\end{aligned}
$$

So we get

$$
\begin{aligned}
\sum_{n=0}^{\infty} d(3 n) q^{n} & =f_{1}^{3} \frac{\varphi^{3}\left(-q^{3}\right) \psi^{3}\left(q^{3}\right)}{\varphi^{4}(-q) \psi^{4}(q)}\left(\frac{1}{w^{2}(q)}-2 q w(q)\right) \\
& =\frac{1}{f_{2}^{2} f_{6}^{3}}\left(\frac{f_{3}^{3}}{f_{1}}\right)^{3}-2 q \frac{f_{6}^{6}}{f_{2}^{5}} .
\end{aligned}
$$


Based on (2.7), we derive that

$$
\sum_{n=0}^{\infty} d(6 n) q^{n}=\frac{f_{2}^{9} f_{3}^{3}}{f_{1}^{8} f_{6}^{3}}+3 q \frac{f_{2} f_{6}^{5}}{f_{1}^{2} f_{3}} \equiv \frac{f_{2}^{9} f_{3}^{3}}{f_{1}^{8} f_{6}^{3}} \equiv f_{1} \quad(\bmod 3) .
$$

Invoking Lemma 2.4, we arrive at that, for any prime $p \geq 5$,

$$
\sum_{n=0}^{\infty} d\left(6\left(p n+\frac{p^{2}-1}{24}\right)\right) q^{n} \equiv(-1)^{\frac{ \pm p-1}{6}} f\left(-q^{p}\right) \quad(\bmod 3),
$$

and

$$
\sum_{n=0}^{\infty} d\left(6\left(p^{2} n+\frac{p^{2}-1}{24}\right)\right) q^{n} \equiv(-1)^{\frac{ \pm p-1}{6}} f(-q) \quad(\bmod 3) .
$$

Furthermore, we have

$$
\sum_{n=0}^{\infty} d\left(6\left(p^{2}\left(p n+\frac{p^{2}-1}{24}\right)+\frac{p^{2}-1}{24}\right)\right) q^{n} \equiv f\left(-q^{p}\right) \quad(\bmod 3) .
$$

Namely,

$$
\sum_{n=0}^{\infty} d\left(6 p^{3} n+\frac{p^{4}-1}{4}\right) q^{n} \equiv f\left(-q^{p}\right) \quad(\bmod 3) .
$$

Thus, by induction on $\alpha$, we derive that, for $\alpha \geq 1$,

$$
\sum_{n=0}^{\infty} d\left(6 p^{2 \alpha-1} n+\frac{p^{2 \alpha}-1}{4}\right) q^{n} \equiv(-1)^{\alpha\left(\frac{ \pm p-1}{6}\right)} f\left(-q^{p}\right) \quad(\bmod 3) .
$$

This yields that, for $j=1,2, \cdots, p-1$,

$$
d\left(6 p^{2 \alpha-1}(p n+j)+\frac{p^{2 \alpha}-1}{4}\right) \equiv 0 \quad(\bmod 3),
$$

which implies (5.2).

Theorem 5.3. For $n \geq 0, \alpha \geq 1$, and prime $p \geq 5$,

$$
d\left(6 p^{2 \alpha} n+\frac{(24 j+9 p) p^{2 \alpha-1}-1}{4}\right) \equiv 0 \quad(\bmod 9),
$$

where $j=1,2, \cdots, p-1$.

Proof. Extracting terms involving $q^{3 n+2}$ and replace $q^{3}$ by $q$ in (5.3), then we derive that

$$
\sum_{n=0}^{\infty} d(3 n+2) q^{n}=\frac{3 f_{3}^{3} f_{6}^{3}}{\varphi(-q) \psi(q) f_{2}^{3}}=\frac{3 f_{3}^{3} f_{6}^{3}}{f_{1} f_{2}^{4}} .
$$

It follows by (2.7) that,

$$
\sum_{n=0}^{\infty} d(3 n+2) q^{n}=3 \frac{f_{3}^{3} f_{6}^{3}}{f_{1} f_{2}^{4}}=3 \frac{f_{4}^{3} f_{6}^{5}}{f_{2}^{6} f_{12}}+3 q \frac{f_{6}^{3} f_{12}^{3}}{f_{2}^{4} f_{4}} .
$$

Hence,

$$
\sum_{n=0}^{\infty} d(6 n+2) q^{n}=3 \frac{f_{2}^{3} f_{3}^{5}}{f_{1}^{6} f_{6}} \equiv 3 f_{9} \quad(\bmod 9) .
$$


In view of Lemma 2.4 , for any prime $p \geq 5$, we deduce that

$$
\sum_{n=0}^{\infty} d\left(6\left(p n+\frac{3\left(p^{2}-1\right)}{8}\right)+2\right) q^{n} \equiv 3(-1)^{\frac{ \pm p-1}{6}} f\left(-q^{9 p}\right) \quad(\bmod 9),
$$

and

$$
\sum_{n=0}^{\infty} d\left(6\left(p^{2} n+\frac{3\left(p^{2}-1\right)}{8}\right)+2\right) q^{n} \equiv 3(-1)^{\frac{ \pm p-1}{6}} f\left(-q^{9}\right) \quad(\bmod 9) .
$$

Moreover,

$$
\sum_{n=0}^{\infty} d\left(6\left(p^{3} n+\frac{3\left(p^{4}-1\right)}{8}\right)+2\right) q^{n} \equiv 3 f\left(-q^{9 p}\right) \quad(\bmod 9) .
$$

Hence, by induction on $\alpha \geq 1$, we arrive at,

$$
\sum_{n=0}^{\infty} d\left(6\left(p^{2 \alpha-1} n+\frac{3\left(p^{2 \alpha}-1\right)}{8}\right)+2\right) q^{n} \equiv 3(-1)^{\alpha\left(\frac{ \pm p-1}{6}\right)} f\left(-q^{9 p}\right) \quad(\bmod 9),
$$

which implies that for $j=1,2, \cdots, p-1$,

$$
d\left(6\left(p^{2 \alpha-1}(p n+j)+\frac{3\left(p^{2 \alpha}-1\right)}{8}\right)+2\right) \equiv 0 \quad(\bmod 9) .
$$

This leads to (5.4).

Theorem 5.4. For $n \geq 0$, we have

$$
d(45 n+t) \equiv 0 \quad(\bmod 5),
$$

where $t=17$ and 35 .

Proof. From (5.5), we have

$$
\sum_{n=0}^{\infty} d(3 n+2) q^{n}=\frac{3 f_{3}^{3} f_{6}^{3}}{\varphi(-q) \psi(q) f_{2}^{3}} .
$$

Again by (2.8), (2.9) and (2.11), we have

$$
\sum_{n=0}^{\infty} d(9 n+8) q^{n}=f_{2} \cdot H
$$

where

$$
\begin{aligned}
H=( & \left.\frac{9 f_{3}^{9} f_{4} f_{6}^{9}}{f_{1}^{3} f_{2}^{13} f_{12}^{3}}+\frac{9 f_{3}^{3} f_{4}^{2} f_{6}^{18}}{f_{1} f_{2}^{16} f_{12}^{6}}\right)+q\left(\frac{27 f_{3}^{6} f_{6}^{9}}{f_{1}^{2} f_{2}^{13}}-\frac{18 f_{4} f_{6}^{18}}{f_{2}^{16} f_{12}^{3}}\right) \\
& +q^{2}\left(\frac{36 f_{3}^{9} f_{12}^{6}}{f_{1}^{3} f_{2}^{10} f_{4}^{2}}+\frac{72 f_{3}^{3} f_{6}^{9} f_{12}^{3}}{f_{1} f_{2}^{13} f_{4}}+\frac{108 f_{1} f_{6}^{18}}{f_{2}^{16} f_{3}^{3}}\right) \\
& -q^{3} \frac{72 f_{6}^{9} f_{12}^{6}}{f_{2}^{13} f_{4}^{2}}+q^{4} \frac{144 f_{3}^{3} f_{12}^{12}}{f_{1} f_{2}^{10} f_{4}^{4}} .
\end{aligned}
$$

We next show a surprising congruence.

Lemma 5.5. It holds that

$$
H \equiv 3 \frac{f_{15}^{3}}{f_{5} f_{10}^{2}} \quad(\bmod 5) .
$$


Proof of Lemma 5.5. To prove (5.7), it suffices to show

$$
H-3 \frac{f_{3}^{15}}{f_{1}^{5} f_{2}^{10}} \equiv 0 \quad(\bmod 5)
$$

or equivalently,

$$
\left(H-3 \frac{f_{3}^{15}}{f_{1}^{5} f_{2}^{10}}\right) \frac{f_{1}^{5} f_{3} f_{4}^{10} f_{6}^{10}}{f_{2}^{4} f_{12}^{6}} \equiv 0 \quad(\bmod 5),
$$

since $\frac{f_{1}^{5} f_{3} f_{4}^{10} f_{6}^{10}}{f_{2}^{4} f_{12}^{6}}$ is invertible in the ring $\mathbb{Z} / 5 \mathbb{Z}[[q]]$. According to Lemma 2.5, it becomes

$$
\frac{15 p^{2}(1-p)(1+p)^{5}(2+p)^{2}\left(2+5 p+12 p^{2}+5 p^{3}+2 p^{4}\right) k^{8}}{32 q^{2}(1+2 p)} \equiv 0 \quad(\bmod 5) .
$$

Lemma 5.5 follows obviously.

We know from Lemma 5.5 that

$$
\sum_{n=0}^{\infty} d(9 n+8) q^{n} \equiv 3 f_{2} \frac{f_{15}^{3}}{f_{5} f_{10}^{2}} \quad(\bmod 5)
$$

Since $f_{2}=\left(q^{2} ; q^{2}\right)_{\infty}$ contains no terms of the form $q^{5 n+1}$ and $q^{5 n+3}$, we have

$$
d(9(5 n+1)+8)=d(45 n+17) \equiv 0 \quad(\bmod 5),
$$

and

$$
d(9(5 n+3)+8)=d(45 n+35) \equiv 0 \quad(\bmod 5),
$$

which leads to Theorem 5.4.

Corollary 5.6. For $n \geq 0$, we have

$$
d(45 n+t) \equiv 0 \quad(\bmod 15),
$$

where $t=17$ and 35 .

Proof. Again, we know from [15, Theorem 4.2] that

$$
d(3 n+2) \equiv 0 \quad(\bmod 3) .
$$

It indeed follows directly from (5.5). We therefore prove Corollary 5.6 by Theorem 5.4 .

\section{Final remarks}

We end this paper by raising the following congruences.

Question 6.1. We have

$$
\begin{aligned}
c(45 n+21) & \equiv 0 \quad(\bmod 5), \\
c(63 n+t) & \equiv 0 \quad(\bmod 7),
\end{aligned}
$$

where $t=30,48$ and 57 .

Question 6.2. We have

$$
\begin{aligned}
d(45 n+41) & \equiv 0 \quad(\bmod 5), \\
d(63 n+t) & \equiv 0 \quad(\bmod 7),
\end{aligned}
$$

where $t=32,50$ and 59 . 
All these congruences have been verified by the authors using an algorithm due to Radu and Sellers [21]. However, since the modular form proofs are very routine and tedious, we here want to ask if there exist elementary proofs of these congruences.

\section{References}

1. Ş. Alaca and K. S. Williams, The number of representations of a positive integer by certain octonary quadratic forms, Funct. Approx. Comment. Math. 43 (2010), part 1, 45-54.

2. G. E. Andrews, Partitions with short sequences and mock theta functions, Proc. Natl. Acad. Sci. USA 102 (2005), no. 13, 4666-4671.

3. G. E. Andrews, A. Dixit, D. Schultz, and A. J. Yee, Overpartitions related to the mock theta function $\omega(q)$, Preprint (2016). Available at arXiv:1603.04352.

4. G. E. Andrews, A. Dixit, and A. J. Yee, Partitions associated with the Ramanujan/Watson mock theta functions $\omega(q), \nu(q)$ and $\phi(q)$, Res. Number Theory 1 (2015), Art. 19, 25 pp.

5. G. E. Andrews and F. G. Garvan, Ramanujan's "lost" notebook. VI. The mock theta conjectures, Adv. in Math. 73 (1989), no. 2, 242-255.

6. G. E. Andrews, D. Passary, J. Seller, and A. J. Yee, Congruences related to the Ramanujan/Watson mock theta functions $\omega(q)$ and $\nu(q)$, Ramanujan J. 43 (2017), no. 2, 347-357.

7. N. D. Baruah and K. K. Ojah, Some congruences deducible from Ramanujan's cubic continued fraction, Int. J. Number Theory 7 (2011), no. 5, 1331-1343.

8. B. C. Berndt, Ramanujan's notebooks. Part III, Springer-Verlag, New York, 1991. xiv+510 pp.

9. B. C. Berndt and R. A. Rankin, Ramanujan: Letters and commentary, American Mathematical Society, London Mathematical Society, History of Mathematics Series, Vol. 9, 1995, 347 pp.

10. A. Blecher, Geometry for totally symmetric plane partitions (TSPPs) with self-conjugate main diagonal, Util. Math. 88 (2012), 223-235.

11. H.-C. Chan, Ramanujan's cubic continued fraction and an analog of his "most beautiful identity", Int. J. Number Theory 6 (2010), no. 3, 673-680.

12. H.-C. Chan, Ramanujan's cubic continued fraction and Ramanujan type congruences for a certain partition function, Int. J. Number Theory 6 (2010), no. 4, 819-834.

13. S. Chern, Congruences for 1-shell totally symmetric plane partitions, Integers 17 (2017), Paper No. A21, 7 pp.

14. Y.-S. Choi, The basic bilateral hypergeometric series and the mock theta functions, Ramanujan J. 24 (2011), no. 3, 345-386.

15. Y.-S. Choi and B. Kim, Partition identities from third and sixth order mock theta functions, European J. Combin. 33 (2012), no. 8, 1739-1754.

16. S.-P. Cui and N. S. S. Gu, Arithmetic properties of $\ell$-regular partitions, Adv. in Appl. Math. 51 (2013), no. 4, 507-523.

17. F. Garvan, D. Kim, and D. Stanton, Cranks and t-cores, Invent. Math. 101 (1990), no. 1, $1-17$.

18. G. Gasper and M. Rahman, Basic hypergeometric series. Second edition, Encyclopedia of Mathematics and its Applications, 96. Cambridge University Press, Cambridge, 2004. xxvi+428 pp.

19. M. D. Hirschhorn and J. A. Sellers, Arithmetic properties of 1-shell totally symmetric plane partitions, Bull. Aust. Math. Soc. 89 (2014), no. 3, 473-478.

20. B. Kim, An analog of crank for a certain kind of partition function arising from the cubic continued fraction, Acta Arith. 148 (2011), no. 1, 1-19.

21. S. Radu and J. A. Sellers, Congruence properties modulo 5 and 7 for the pod function, Int. J. Number Theory 7 (2011), no. 8, 2249-2259.

22. L.-C. Shen, On the modular equations of degree 3, Proc. Amer. Math. Soc. 122 (1994), no. 4, 1101-1114.

23. L. Wang, Arithmetic identities and congruences for partition triples with 3-cores, Int. J. Number Theory 12 (2016), no. 4, 995-1010.

24. E. X. W. Xia, A new congruence modulo 25 for 1-shell totally symmetric plane partitions, Bull. Aust. Math. Soc. 91 (2015), no. 1, 41-46.

25. E. X. W. Xia and O. X. M. Yao, Analogues of Ramanujan's partition identities, Ramanujan J. 31 (2013), no. 3, 373-396. 
26. O. X. M. Yao, New infinite families of congruences modulo 4 and 8 for 1-shell totally symmetric plane partitions, Bull. Aust. Math. Soc. 90 (2014), no. 1, 37-46.

(S. Chern) Department of Mathematics, The Pennsylvania State University, UniverSITY PARK, PA 16802, USA

E-mail address: shanechern@psu.edu; chenxiaohang92@gmail.com

(L.-J. Hao) Center for Combinatorics, LPMC, Nankai University, Tianjin 300071, P.R. CHINA

E-mail address: haolijun152@163.com 